\title{
File Annotation and Sharing on Low end Devices in PAN
}

\author{
Megha B. Jadhav \\ M.B.E.Society's College of Engineering, Ambajogai. \\ Maharashtra.
}

\author{
B. M. Patil \\ M.B.E.Society's College of Engineering, Ambajogai. \\ Maharashtra.
}

\begin{abstract}
Fast development in low end devices permits so many extra aspects including huge storage capacity, sharing of data, formation of network, etc. User can use these aspects very efficiently. When data size is limited searching a particular file can done easily but difficulty comes when data size increases and it involves large collection of mobile nodes. In that case arrangement of data i.e. data organization and management become irritating work. However file system of devices itself provides a way to store similar files separately; still it becomes irritating when number of files increases. Even poor UI (User Interface) increases more difficulty in case of file retrieval. This paper presents model that allows user to annotate files and to search a file of interest on mobile devices. File is annotated using their spatial, common and temporal circumstance which are extracted from underlining operating system and used as a metadata which is used for searching a file of interest. The file search can be achieved on mobile itself or on other mobile also those are connected in Personal Area Network (PAN) using Bluetooth. The proposed model allows file annotation, file search, creating PAN (Personal Area Network), file sharing, etc.
\end{abstract}

\section{General Terms}

File Annotation System, Meta data, Parsing.

\section{Keywords}

File Annotation, Bluetooth, PAN, kXML parser.

\section{INTRODUCTION}

Importance of mobile phone cannot be neglected as now a day's it plays necessary role to stay alive with day by day life. As the technology advances, mobile phones are available with elevated storage capacity and supplementary features like camera, additional communication interface, etc. However difficulty generated as user start using such features and it generates a huge files. From the huge collection, it becomes complicated to memorize the file name and its contents. The default file system available with device provide the way to store in separate folder but still it doesn't help due to naming convention used. Usually when image is captured using mobile camera, it gets stored with default file name like img000, img001, etc, which are not descriptive sufficient to memorize. To improve search technique file annotation is implemented which annotates the file with its vital attribute from the underlying file system of the mobile phone, and sues vital attribute information as the annotation tags and parse those tags using $\mathrm{kXML}$ parser to store in XML form. The proposed model annotates file with three fundamental attribute and two additional tags are also added .XML is used to parse the XML data. File can be searched through any attribute on device itself or other connected device within Personal Area Network (PAN) by the use of Bluetooth. Proposed model is implemented in Java Micro Edition (J2ME) [1].

\section{LITERATURE SURVEY}

Technical progress in low end devices has been significant in recent years which provide mobile phones enhanced with so many advance features. Recent research exposed various techniques between which semantic file aware system is the most approved alternatives to traditional hierarchical file system. The data model was proposed to represent semantic information in file system which provides two main features: extensibility and handling schema valuation. Similarly, various procedures were proposed for integrating attribute in $[3,4]$. Efforts have been made to expand file annotation on mobile devices but it show complication due to resource limitations.

In paper [5] W.Viananl, J.B. Filho2, J.Gensel, M.Villanova Oliver, H.Martin focused on Context Photo Ontology which makes the use of annotation that allows the development of better management and retrieval for images. In the context of Semantic Web, the use of ontologies for annotation representation is more suitable for making the content machine understandable.

Soules CAN, G.R Ganger [6] found that when data set associated with a user grow up, organizing that information turn out to be more complicated. As the chain of command provides valuable aspect, they cannot manage easily. A new flexible attribute-based naming scheme is required to manage large set of data.

B.Gopal and U. Manber [7] offered innovative file system that provides content-based and name-based access files at a time. The proposed model allows both methods to be used at any time, thus gives advantages of both. F.monaghan, O'Sullivan [8] presented Web service and ontology based image annotation approach to annotate files. A.Girgensohn, J. Adcock, M. Cooper, J. Foote, L.Wilcox [9] in his paper stated that users could simply gather number of images. The goal was to make arranging and browsing of images easy and quick, which provides scalability to huge data.

Pratibha Singh, Dipesh Sharma [10] presented a model to make communication within Personal Area Network(PAN). Communication in this model is made via Bluetooth. Bluetooth is a wireless technology standard for exchanging data over short distances from fixed and mobile devices, creating personal area networks (PAN) with high levels of security.

In all mechanism, either annotation process or retrieval process will not be viable in case of network failure. A new mechanism is needed to annotate all type of files on mobile devices and provide higher success rate for searching file of interest. 


\section{JAVA 2 MICRO EDITION (J2ME)}

$\mathrm{J} 2 \mathrm{ME}$ is one of the common platforms used to mobile phone application development. This platform was developed by Sun Microsystems in 1999 which offers a strong environment for application developers. Java Community Process (JCP) is process to develop standards for Java technology and they defined various configurations and profiles to achieve diverse requirements of application developers. J2ME uses a compact version Kilo Virtual Machine (KVM) to make the entire architecture more modular and scalable. Configuration is the run-time environment and classes operating on a device while profile is the set of domain specific classes to implement relevant features on a related group of low end devices. Configuration layer has two parts namely, Connected Limited Device Configuration (CLDC) and Connected Device Configuration (CDC). The Profile layer consists of five different profiles, in which Mobile Information Device Profile (MIDP) is usually used profile with LDC as it contains classes to deal with networking, storage and user interface capabilities. MIDlets are MIDP applications and the software that implements MIDP running in KVM. We used the similar combination to execute our proposed framework using the core classes for file system, Bluetooth and graphical user interface (GUI). The meta-data is required to be processed and manipulated in XML format but its parser tends to be bulky for resource limited devices. XML is a meta-markup language which was approved by $\mathrm{W} 3 \mathrm{C}$ and has become universally acknowledged specification for exchanging documents and data across applications and platforms. kXML parser is used to parse XML data, which is a lighter and compact version of XML parser particularly designed for low-end devices and is exclusively used on J2ME platform [11][12].
Three types of parser includes model, push and pull parser. Model parser reads the whole document and then creates a presentation in memory and sometime requires vast memory depending on the size of the XML document [13]. Push parsers process data definitions prior to the creation of tree structure in memory, while pull parser reads the data first before it starts parsing. Pull parser uses recursive functions to create document tree and therefore are more appropriate for handheld devices.

\subsection{XML Parser \& XML Data}

XML Parser is used to collect the meta-data of the file to be annotated from the annotation process during annotation and push it into a centralized XML Data. Conversely, it would retrieve the meta-data of the files which match the search key and criteria during searching from the XML Data .

\subsubsection{Why XML?}

There are some specific advantages of using XML:

- Platform Independent

- $\quad$ Easy to understand

- Self describing data

- $\quad$ Extracted GUI

\section{PROPOSED MODEL}

This paper presents a model that allows annotation of all type of file, searching technique for retrieving files on mobile devices with minimum efforts using different type of attribute, sees console, creation a PAN via Bluetooth and sharing of data within PAN. Fig. 1 shows the proposed model architecture[14].

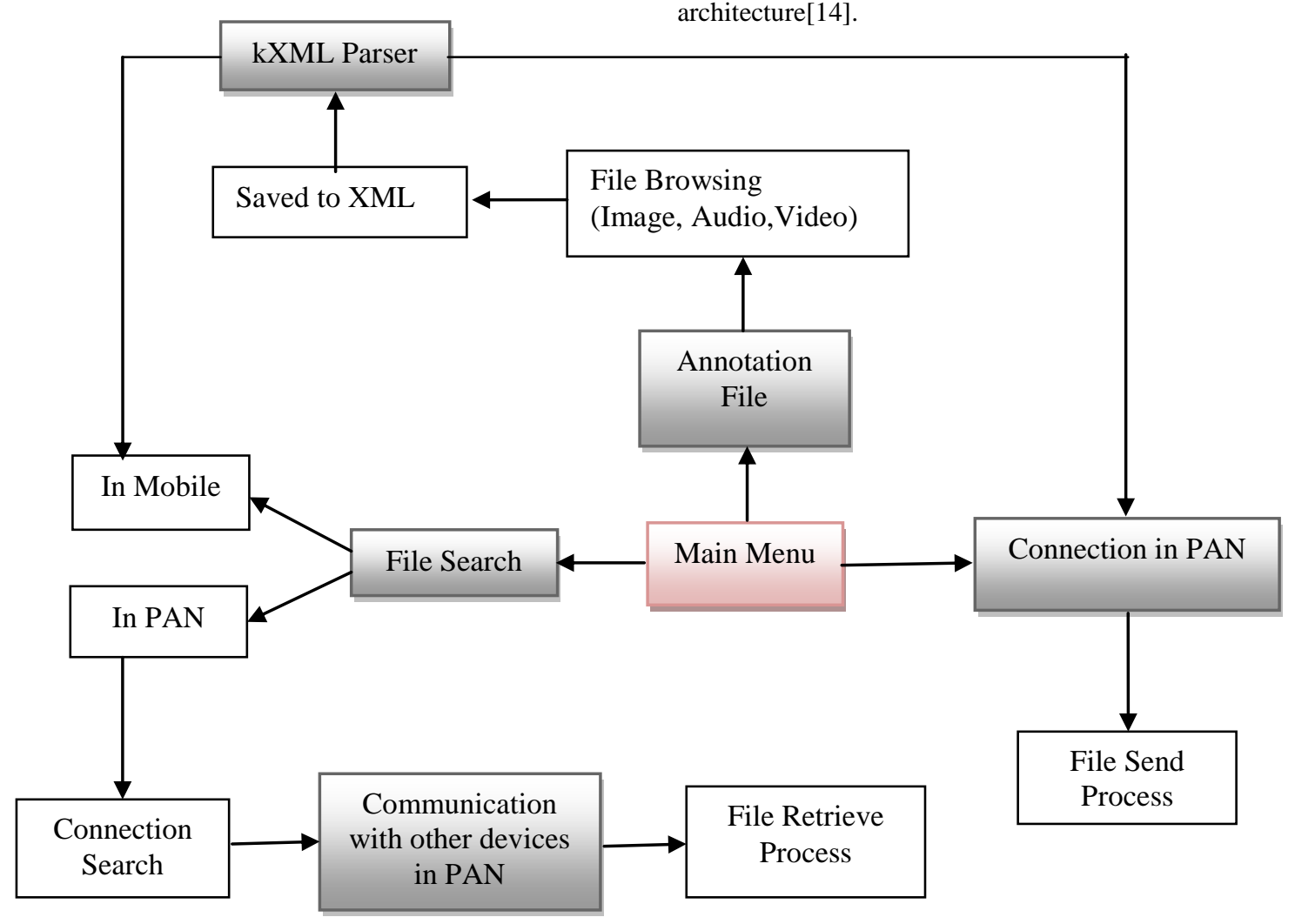

Fig 1: Proposed Model Architecture 
In a scenario, a user had taken few pictures with his/her friends on birthday party using mobile phone camera which were saved by default naming like image000, image001, image002, etc. After few days user want a particular image but unable to find out required one. She $/ \mathrm{He}$ also forgot whose mobile was used to take image because most of the member took images at that party. To search for the required image she /he has to look around all image file till required image found and need to repeat browsing on other friends mobile devises also those took images on that party. For the same, the model presented in this paper gives easy way to retrieve file using advanced search method. With the help of fundamental attributes of file user can easily retrieve the file, even though file is not annotated with optional tags. If required file is not on her mobile phone, it can be searched on all other mobile also by forming PAN via Bluetooth [14].

\subsection{File Annotation}

This module interacts with the underlying operating system of device to get the attributes of files. These attributes are used as annotation tags for each corresponding file. This annotation is of two types:

\subsubsection{Automatic Annotation:}

In Automatic meta-data part, files are annotated automatically with three basic attributes which includes file-name, file-size and date-of-creation.

\subsubsection{Optional Annotation:}

In this annotation two additional tags can be appended through Optional meta-data part. These two optional tags are Keyword and Description, which users have to type in for each file. Annotation is a onetime process but it can be edited or updated anytime for any stored file on the device. All attribute are parsed and stored in XML format. Fig. 2 shows the file annotation process.

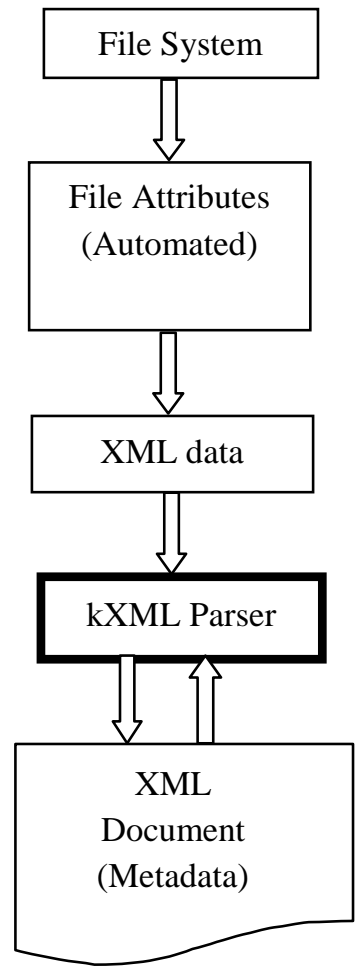

Annotation Process is follows:

1.) The 'Automatic' meta-data is taken from the attributes of the file being annotated.

2.) This 'Automatic' meta-data is combined with the User-defined data, i.e., 'Optional' meta-data.

3.) Finally, the final meta-data is pushed into an XML Document in the form of XML Data using the XML Parser.

4.) Steps 1-3 can be repeated over and over, till the user wishes to annotate a file.

\subsection{Search Process}

Search process nearly interacts with all other process included in a model and plays essential role in proposed model. To search information about the required file this process uses kXML parser for processing XML document. As file is annotated with Automated and Optional tags, search can be performed with different available attribute as shown in Fig. 6 , however optional tags can be used to search a file only if file is annotated using optional tag. If search is performed within PAN, search process uses Bluetooth to form a network. Fig. 3 shows attributes stored in XML file as meta-data. All devices within PAN also parse their local XML documents and send back the search results. If file name is found, it is shared and sends with requesting device.

If the user specifies the search criteria for the search as FileSize, then, keeping in mind the human impreciseness, a tolerance range of some units is put up to achieve a successful search. The unit for the tolerance is based on the size type as in the search criteria, i.e., $\mathrm{KB}$ or $\mathrm{MB}$, with $+/-100$ for $\mathrm{KB}$ and $+/-10$ for MB.

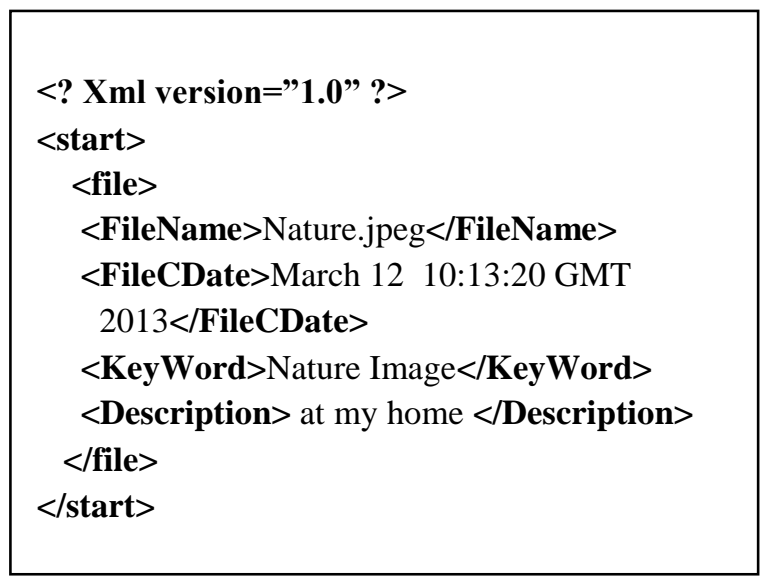

Fig 3: Annotation Tags

\subsection{Bluetooth}

Bluetooth is a wireless technology standard for exchanging data over short distances and it is available in almost all new mobile devices. With the help of Bluetooth proposed model form PAN. PAN allows sharing and managing of data within a formed network. The Bluetooth protocol stack is standardized in JSR-82 which gives good control for stack initialization, device management, device discovery, service discovery, and communication. Service Discovery Protocol (SDP) is used to discover the nearby devices and files shared by other users in the network [14]. 
The implemented MIDlet's main screen is shown in Fig. 4. Fig. 5 shows file to be annotated with its attribute, Fig. 6 shows meta-data for annotated file can be viewed as a list.

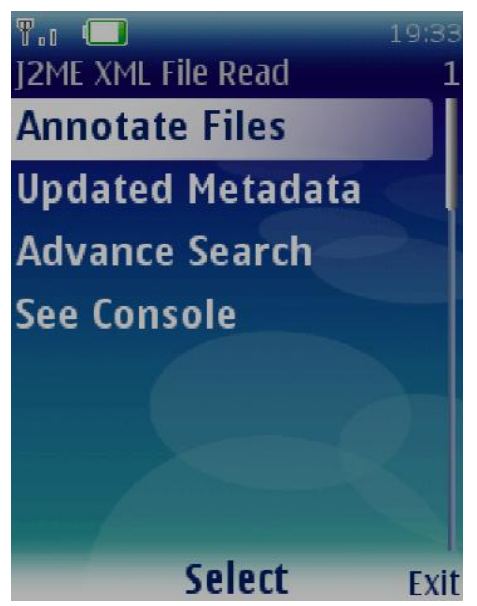

Fig 4: Main Screen

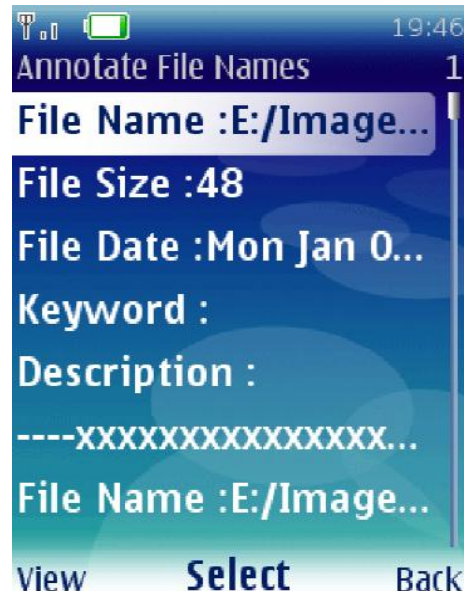

Fig 6: Meta-data

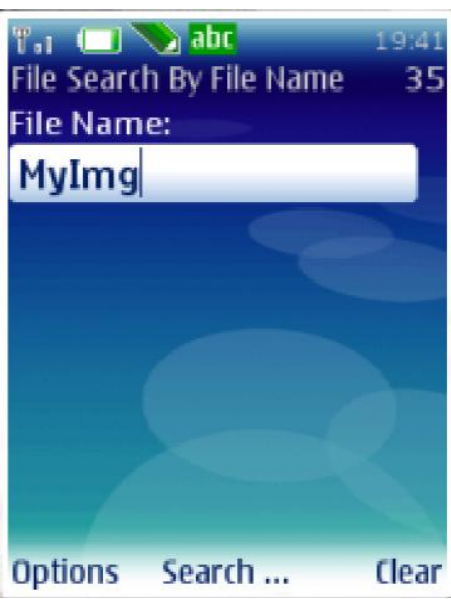

Fig 8: Search by File Name
File search can be performed using different attributes as shown in Fig.7, Fig 8. Shows the way file is searched using file name, Fig 9. Shows search result.

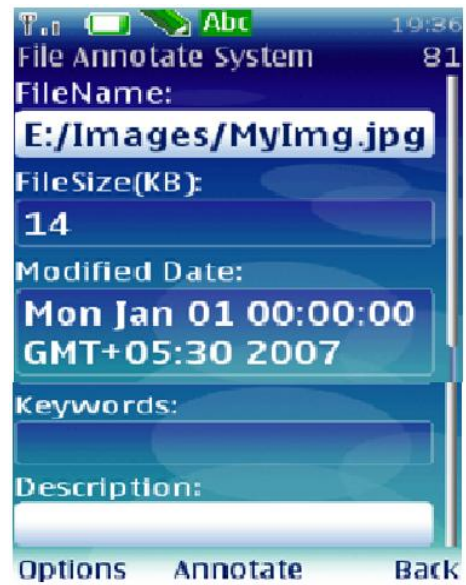

Fig 5: File Annotation

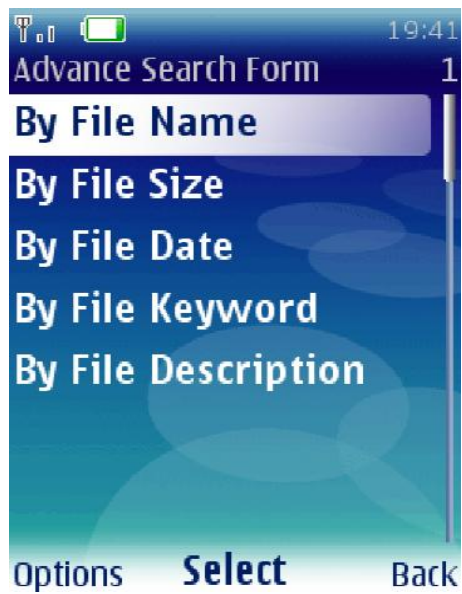

Fig 7: Search Attributes

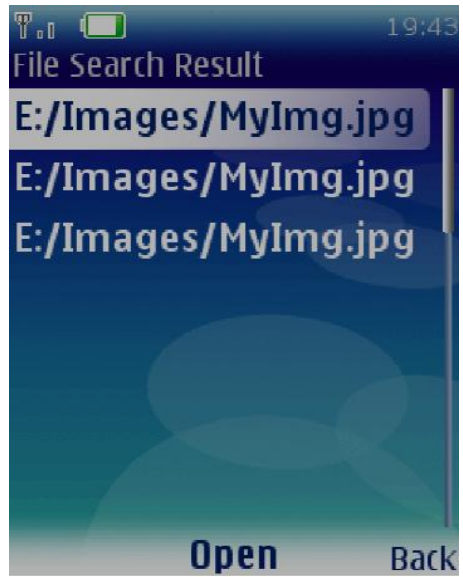

Fig 9: Search Results 


\section{RESULTS}

To calculate the importance of meta-data in our proposed model, we created two set of tests that include 20 search queries. For the first test, all files were annotated and the optional tags were not added and for the second test, files were only annotated and additional tags also added. Result shows that success rate is $95 \%$ for the set which was fully annotated and $80 \%$ for the files that were annotated without optional tags.

Table 1. Query types and Results

\begin{tabular}{|c|l|c|}
\hline $\begin{array}{c}\text { Number of } \\
\text { Query }\end{array}$ & \multicolumn{1}{|c|}{ Attributes } & $\begin{array}{c}\text { Result of } \\
\text { success }\end{array}$ \\
\hline 20 & $\begin{array}{l}\text { File Name, File Size, } \\
\text { Date of creation }\end{array}$ & 19 \\
\hline 20 & $\begin{array}{l}\text { File Name, File Size, } \\
\text { Date of creation, } \\
\text { Keyword, Description }\end{array}$ & 16 \\
\hline
\end{tabular}

The significance of user-typed additional tags can be measured by the difference between two sets of results, which is $15 \%$ in this case.

\section{CONCLUSIONS}

This paper presents a model that improves semantic file annotation and file retrieving in on mobile phones using PAN. The model is implemented as full featured MIDlet and adding Optional tags as meta-data surely enhance the search ability to retrieve files based on keywords and description.

The importance of adding optional tags as meta-data is analyzed which surely enhances the search ability. Addition to the current work, we plan to attempt improve search result by adding ontology to the stored meta-data to add the semantic based search to the existing framework. For this purpose, a framework is required to provide programmatic environment to support Ontology Web Language and Resource Description Framework (OWL/RDF) along with computationally lighter reasoned for resource limited devices. Various techniques will also be analyzed to create and update ontology and its role in file search.

\section{REFERENCES}

[1] Sadaqat Jan, Maozhen Li, Ghaidaa Al-Sultany and Hamed Al-Raweshidy "File Annotation and Sharing on Low-End Mobile Devices", Seventh International Conference on Fuzzy Systems and Knowledge Discovery(FSKD),2010.
[2] D.P.Pandit, L.M.R.J. Lobo “ File Annotation on Mobile Devices", International Journal of Engineering Research \& Technology (IJERT), October - 2012.

[3] B. Gopal and U. Manber. Integrating content-based access mechanisms with hierarchical file systems. Symposium on Operating Systems Design and Implementation, pages 265-278. ACM, 1999.

[4] S. Sechrest and M. McClennen. Blending hierarchical and attributebased file naming. International Conference on Distributed Computing Systems, pages 572-580, 1992.

[5] W. Viana1, J. B. Filho2, J. Gensel, M. Villanova- Oliver, H. Martin, "A Semantic Approach and a Web Tool for Contextual Annotation of Photos Using Camera Phones",9th Workshop on Hot Topics in Operating Systems (HotOS IX).May 18-21, 2003.

[6] Soules CAN, G.R.Ganger "Why can't I find my files? New methods for automating attribute assignment", HotOS IX: the 9th workshop on hot topics in operating systems. USENIX Association,May 2003.

[7] B. Gopal and U. Manber. "Integrating content-based access mechanisms with hierarchical file systems", Symposium on Operating system.

[8] F. Monaghan, O'Sullivan, “Automating Photo Annotation using Services and Ontologies", Mobile Services and Ontologies Workshop, 2006.

[9] A. Girgensohn, J. Adcock, M. Cooper, J. Foote. And L.Wilcox. "Simplifying the Management of Large Photo Collections", INTERACT. IOS Press 2003.

[10] Pratibha Singh, Dipesh Sharma, "Study of Bluetooth wireless technology using java", indian Journal of Computer Science and Engineering. Vol. 2, No. 3, JunJul 2011.

[11] "Parsing XML in J2ME”, by Jonathan Knudsen.

[12] James White, "An introduction to Java 2 micro edition (J2ME)", Reference Book. [9] Sun Microsystems, "Mobile Information Device Profile (JSR-37)" JCP Specification, Java 2 Platform, Micro Edition.

[13] "Connected Limited Device Configuration (CDLC)" Specification, Java ${ }^{\mathrm{TM}} 2$ Platform, MicroEdition(J2ME).

[14] Miss. Megha B. Jadhav*, Dr. B.M Patil "File Annotation and Sharing on Mobile Devices in PAN via Bluetooth" International Journal of Scientific and Research Publications, Volume 3, Issue 6, June 2013 ISSN 22503153. 